\title{
Web Based Tools for Signals and Systems Course
}

\author{
doi:10.3991/ijoe.v5i2.896 \\ D. Taskovski, V. Kitanovski and S. Bogdanova \\ University Ss Cyril and Methodius, Skopje, R. Macedonia
}

\begin{abstract}
In this paper we present web-based tools that visualize standard concepts (convolution of analog and discrete signals, pole-zero diagram, Fourier series, sampling and reconstruction) in a classical Signal and Systems course. The tools can be used by a teacher as a teaching aid to explain basic concepts in Signal and Systems course. They can also be used by students as a learning aid. They are easy for use and can be used in different locations using different operating systems.
\end{abstract}

Index Terms-web-based tools, interactive, signals and systems

\section{INTRODUCTION}

The use of new computing technologies in the educational process is becoming more and more common. These technologies can help students visualize certain concepts that are difficult to understand in classical lectures. Several tools that are used for teaching/learning Signals and Systems can be found on web. Different software packages (Matlab, Java, Octave) are used for the tools development. Among tools developed in Matlab, tools presented in [1],[2] are very intuitive and easy for use. However, the tools developed using Matlab can not be run inside a web page. This is a serious drawback as elearning is increasing in importance in the teaching/learning process.

There are two main reasons using Java for web-based tool development. First, it is very easy to place Java programs on the web making them available to a wide audience. Also, Java is platform independent, which means that a code is compiled only once and can be executed on every computing platform, without the need of precompiling or installing other platform-specific software. And second, Java is free and is incorporated into the most popular web browsers, so users don't have to buy or install any software. On the other hand, Matlab is relatively expensive and it requires a copy of the interface software to be installed on every user's local machine. This is a very important design issue, especially when the main target groups for using the tools are students. They will be more likely to use a tool which is freely available rather than one which is costly.

There have been several isolated attempts to develop JAVA tools for Signal and Systems education that can be used on the Internet for e-learning [3][4][5]. However, taking into account that most of them were written several years ago, when the development of GUIs in JAVA wasn't simple, the functionality of these tools lags behind their MATLAB counterparts. The interaction, which is an important element in the learning process, is minimal in these tools.

In this paper we present new web-based tools for visualization of basic concepts (convolution of analog and discrete signals, pole-zero diagram, sampling and reconstruction) covered in a classical Signal and Systems course. The tools can be used by the teacher as a teaching aid to complement the explanation of related concepts to students in lecture. With the possibility of changing signals and basic parameters, these tools can help students to achieve deeper understanding of the material and even to check their handwritten work.

\section{AN OVERVIEW OF THE COURSE}

The material addressed in the Signals and Systems course is quite typical of introductory signal and system analysis courses in curricula that cover continuous-time analysis separately from discrete-time analysis. Such a course is followed by a fundamental DSP course. The main topics of the course are:

- Description and classification of signals and systems (analogue and digital);

- Time domain analysis - impulse response and convolution (analogue and digital);

- Frequency domain analysis of periodic signals;

- The Fourier transform and some of its applications;

- The Laplace transform and some of its applications

During a 14-week term, the course includes three hours of lecturing and two hours of tutorials and has no formal laboratory component. Students are expected to invest about 15 to 20 hours per week, including class time and homework, into the course. The exam is written and consists of problem solving and the main effort of the most students is focused on problem solving techniques without understanding the main concepts. This approach results in memorizing particular problems to pass the exam instead of acquiring real knowledge and skills.

As one of the fundamental courses in engineering education, this course is the bedrock for all other courses in modern engendering curricula. The material is largely abstract and highly conceptual, and requires strong mathematical skills as well as comprehension of the meaning behind the mathematics. In essence, the students have to be introduced to the notion of thinking and working in the frequency domain. Although this is a common and important paradigm for electrical engineers, students typically struggle with the mathematical techniques, the conceptual understandings, or both. Furthermore, there are a number of techniques, such as convolution, the determination of Fourier series and their spectra, and the determination of Fourier transforms and their spectra, which demand considerable time and practice to master and require a good understanding of appropriate mathematical concepts like calculus, differential equations, and complex analysis. Finally, modern treatment of this material requires that students become familiar with mathematical modeling tools such as Matlab. Consequently, the students 
lack interest and motivation for deeper understanding of basic concepts and many perform poorly in the class.

\section{BLENDED LEARNING APPROACH}

One of the available ways to overcome these weaknesses is via blended learning approach. This makes the teaching process more interesting and acceptable for students by taking advantage of new information technologies.

The key problem was to address the increasing student engagement in the educational process and to increase their interest and motivation. The challenge was therefore to reverse trend of student passivity during the lessons making use of the opportunities provided by the new IT technologies familiar to students.

We feel that the face-to-face teaching and learning methods of lecture, tutorial, laboratory, used to support the course should be maintained, but supplemented by strategic use of other technologies as appropriate.

In past three years we have tried several approaches, including:

- Tools for visualization of main theoretical concepts (2006)

- Collaborative learning $(2007,2008)$

- Use of LMS (2008).

The following factors were taken into consideration when developing our blended learning model:

- Motivation of students

- specific attributes of the course content,

- available resources,

- level of faculty competence to develop online educational material,

- The number of students, and their readiness to access, adopt, and effectively utilize online course materials.

As a first step, we have added to traditional teaching and learning methods new web-based tools for visualization of basic concepts in a signal processing course. They help students visualize the basic concepts (convolution of analog and discrete signals, pole-zero diagram, sampling and reconstruction) covered in a classical Signal and Systems course.

The tools serve as a teaching aid to the teacher and complement the explanation of related concepts to students in lecture. By changing sample signals and basic parameters, students can experiment with tools and achieve a deeper understanding of the material. Additionally all tools are complemented by tutorials explaining the main theoretical concepts, and with solved and unsolved examples. This can help students take advantage of the main feature of blended learning: learning at any time and anywhere.

\section{SignALS AND SYSTEMS TOOLS}

\section{A. Convolution of analog and discrete signals}

These tools give graphical visualization of one of the most important and difficult to understand concepts in signal processing - convolution of analog or discrete signals. User can select two signals from the list of predefined signals, and adjust some of the signal's parameters, like amplitude, length or delay. There is also possibility to draw arbitrary signals. In this way, the user has a chance to verify his handwritten work. After selecting or drawing signals, they appear in the first window. Using a slider, user can move the flipped signal. The second window displays the multiplication of the overlapping signals. The integral/sum of the multiplicated signals, which represents the value of the convolution for that particular $t$ (or one sample in the convolution of discrete signals) is given in the third window. The graphical animation that displays the convolving process can be also viewed. A screenshot of the analog convolution tool is shown in Fig. 1.

\section{B. Pole-zero diagram for analog and discrete systems}

Systems are completely determined by the location of poles and zeros of their transfer functions. This tool helps the user to understand the impact of poles and zeros locations on the impulse, magnitude and phase response of the system. The poles and zeros can be interactively added on the $s$ - or the $z$-plane. After adding each pole or zero, the three graphics that show magnitude, phase and impulse response are updated. These three system's responses are updated in real time as the user drags individual poles or zeros. This tool can also demonstrate the influence of the poles and zeros on the system stability. Placing or moving a pole in the right side of the $s$-plane (or outside the unit circle in the $z$-plane) causes plane to change its color, indicating that the system is unstable.

\section{Fourier series of periodic signals}

This tool demonstrates analysis of periodic signals using Fourier series, and synthesis of periodic signals using different number of their Fourier coefficients. User can select one of the following periodic signals: square wave, triangle, sawtooth, sine and cosine wave, and set the signal's period. User can also draw its own periodic signal. Fourier coefficients are showed on two graphs in sine/cosine or magnitude/phase mode. User can see the effect of signal translation on the Fourier coefficients. Using a slider, user can select the number of Fourier coefficients to be used for signal synthesis. It's easy to perceive the Gibbs phenomenon when different number of coefficients for the square signal synthesis is selected. In this way, the concept of signal approximation using Fourier basis functions becomes clearer. A screenshot of this tool is shown in Fig. 2.

\section{Sampling and reconstruction}

In many applications analog signals are first converted into discrete signals using sampling operations. These signals are than processed by digital signal processors, and the obtained signals are converted into analog signals using a reconstruction operation. All these operations and their effects can be analyzed with this demo. The user can select the analog signal from the list of predefined signals. The selected signal and its spectrum appear in the first row. After selecting the sampling frequency the discrete signal and its spectrum appear in the second row. The replicas of the frequency spectrum are shown to visualize the effect of sampling in the frequency domain. If the sampling frequency is bellow the Nyquist rate there will be a considerable amount of aliasing. The shape of the frequency spectrum will be different from that of as can be seen to be a result of adding overlapping replicas of continuous signals' frequency spectrum. 
The effect of different interpolators in the process of signal reconstruction is shown on the third row. The user can notice, for instance, that even first-order interpolation provides an acceptable reconstruction when the sampling frequency is much higher than the Nyquist rate. A screenshot of this tool is shown in Fig. 3.

\section{CONCLUSION}

In this paper web-based tools for visualization of basic concepts in a Signal and Systems course were presented. The tools can be used by a teacher as a teaching aid to explain basic concepts in Signal and Systems course. They can also be used by students as a learning aid. They are easy for use and can be used in different locations using different operating systems. These features make the proposed tools competitive with other web-based tools that already exist and offer an attractive alternative for Signal and Systems education. All tools are freely available at http://dsp.etf.ukim.edu.mk.

\section{ACKNOWLEDGMENT}

This paper is prepared in the framework of the Tempus project "Master studies in DSP based on blended learning approach." This project has been funded with support from the European Commission. This paper reflects the views only of the author, and the Commission cannot be held responsible for any use which may be made of the information contained therein.

\section{REFERENCES}

[1] J. Rosenthal, J. McClellan, "Animations and GUIS for Introductory Engineering Courses," in Proc International Conference on Engineering Education, Oslo, Norway, 2001
[2] U. Rajashekar and A. Bovik, 'Interactive DSP Education Using Matlab Demos," in Proc IEEE Signal Processing Education Workshop, Oct. 15-18, 2000, Hunt, Texas.

[3] J. Shaffer, J. Hamaker, and J. Picone, "Visualization of signal processing concepts," in Proc IEEE ICASSP-98, Seattle, pp. 1853-56, 1998

[4] Chen Lee, H., Crutchfield, S., Hocker, C., Kahn, S., Ma, L., Nesky, M., Rosenbaum, K., Rugh, J, W., Venkataramani, R. and Woo, B., 2000, "Signals Systems Control Demonstrations", http://www.jhu.edu/ signals/, The Johns Hopkins University, U.S.A.

[5] Belkhouja, A., Rabenstein, R., and McLachlan, A., 2000, "Systool", http://www.nt.e-technik.uni-erlangen.de/ anis/systool.html, Telecommunications Institute I, University of Erlangen-Nürnberg, Germany.

\section{AUTHORS}

D. Taskovski is with the Ss Cyril and Methodius University, Faculty of Electrical Engineering and Information Technologies, Department of Electronics, Karpos 2 bb, p.o. box 574, Skopje, Macedonia (e-mail: dtaskov@feit.ukim.edu.mk).

V. Kitanovski is with the Ss Cyril and Methodius University, Faculty of Electrical Engineering and Information Technologies, Department of Electronics, Karpos 2 bb, p.o. box 574, Skopje, Macedonia (e-mail: vlade@feit.ukim.edu.mk).

S. Bogdanova, is with the Ss Cyril and Methodius University, Faculty of Electrical Engineering and Information Technologies, Department of Electronics, Karpos 2 bb, p.o. box 574, Skopje, Macedonia (e-mail: sofija@feit.ukim.edu.mk).

Manuscript received 6 April. Published as submitted by the authors.

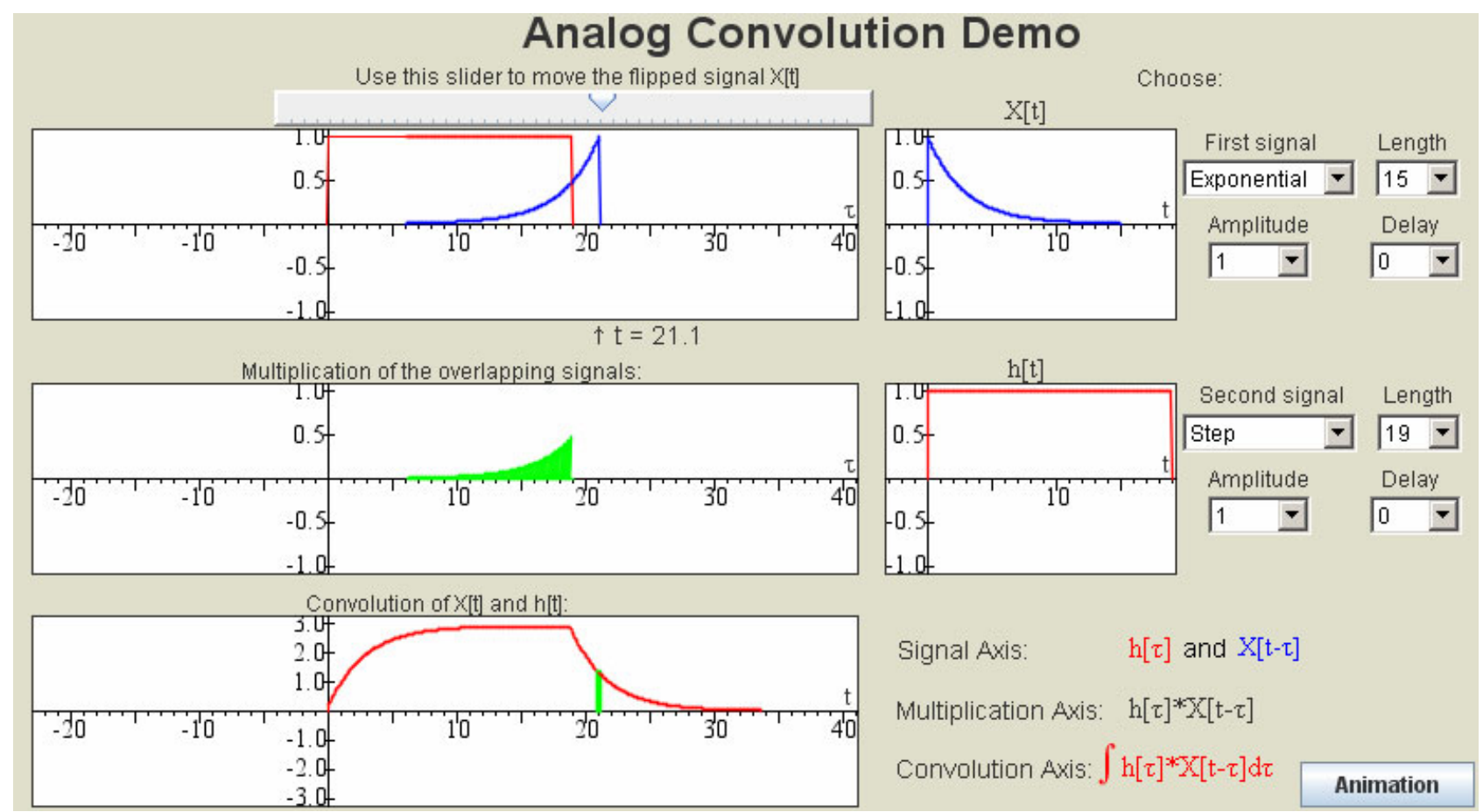

Figure 1. Screenshot of the analog convolution tool 


\section{Fourier Series Demo}

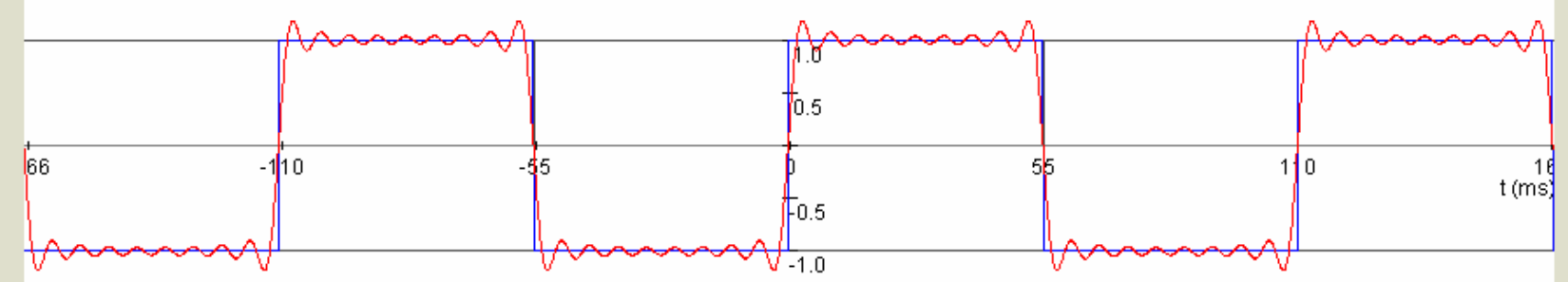

Magnitudes

Phases
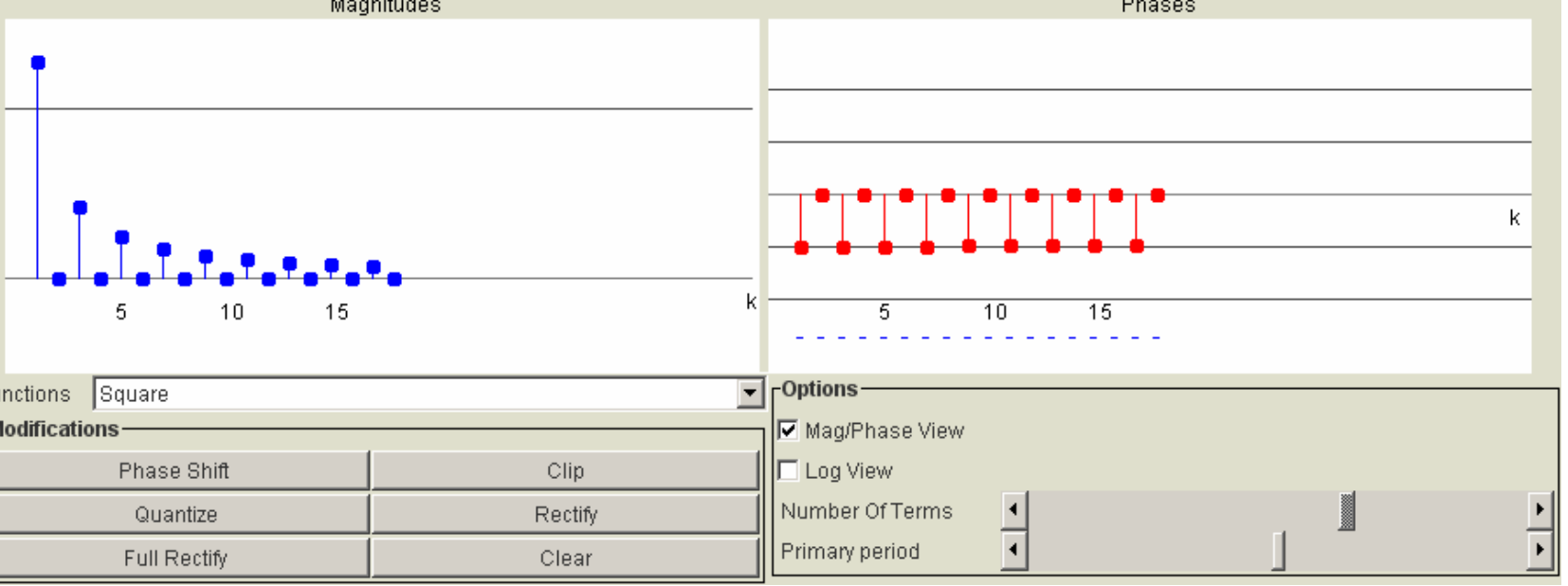

Figure 2. Screenshot of the Pole-Zero tool for analog systems

\section{Sampling and Reconstruction}
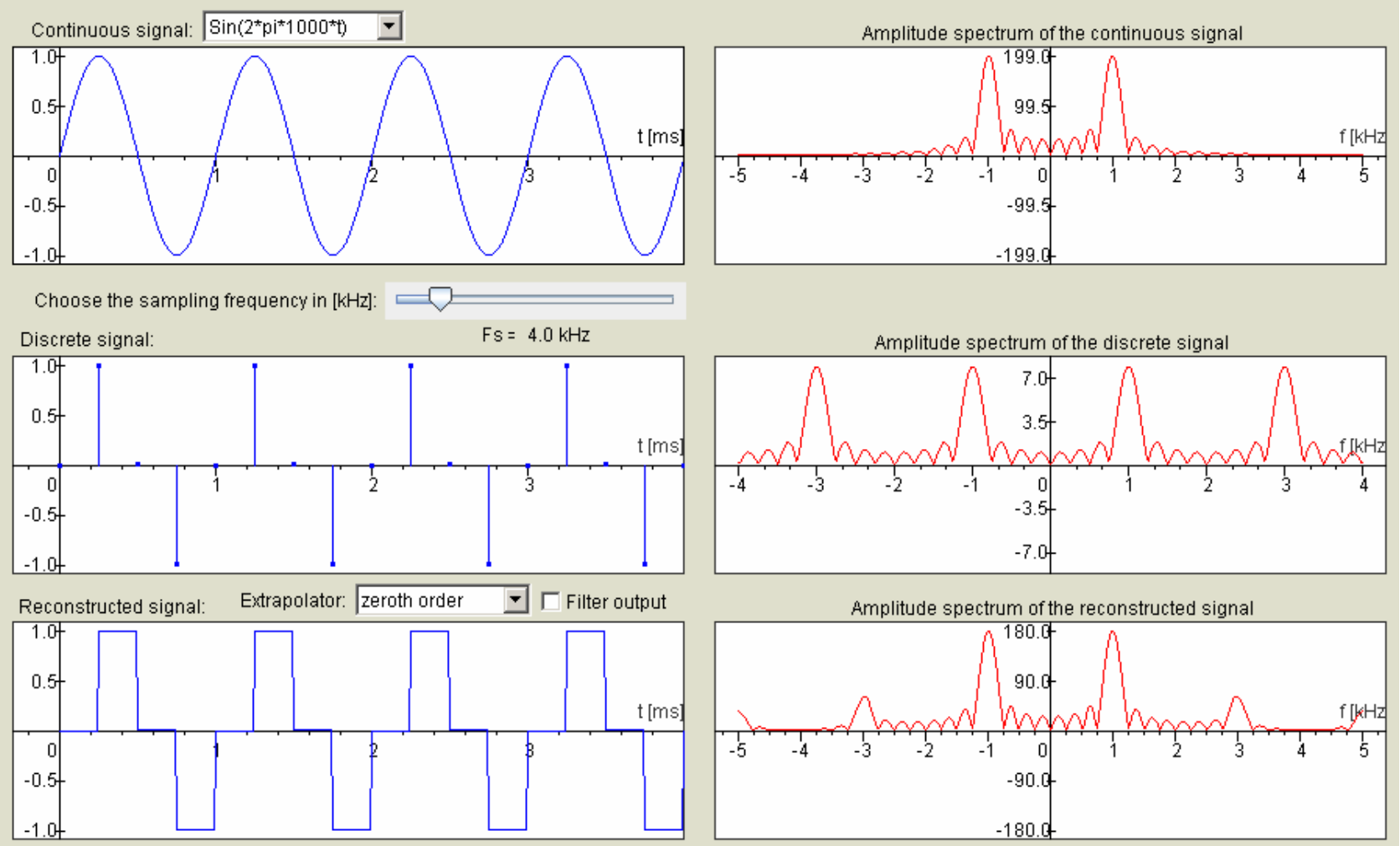

Figure 3. Screenshot of the Sampling and Reconstruction tool 